\title{
Atom interferometry tests of local Lorentz invariance in gravity and electrodynamics
}

\author{
Keng-Yeow Chung \\ Physics Department, National University of Singapore, 2 Science Drive 3, Singapore 117542 \\ Sheng-wey Chiow \\ Physics Department, 382 Via Pueblo Mall, Stanford, California 94305, USA \\ Sven Herrmann \\ ZARM - University of Bremen Am Fallturm/Hochschulring, 28359 Bremen, Germany \\ Steven Chu \\ Department of Physics, University of California, Berkeley, California 94720, USA \\ and Lawrence Berkeley National Laboratory, One Cyclotron Road, Berkeley, California 94720, USA \\ Holger Müller* \\ Department of Physics, University of California, Berkeley, California 94720, USA
}

(Received 12 May 2009; published 6 July 2009)

\begin{abstract}
We present atom-interferometer tests of the local Lorentz invariance of post-Newtonian gravity. An experiment probing for anomalous vertical gravity on Earth, which has already been performed, uses the highest-resolution atomic gravimeter so far. The influence of Lorentz violation in electrodynamics is also taken into account, resulting in combined bounds on Lorentz violation in gravity and electrodynamics. Expressed within the standard model extension or Nordtvedt's anisotropic universe model, we limit 12 linear combinations of seven coefficients for Lorentz violation at the part per billion level, from which we derive limits on six coefficients (and seven when taking into account additional data from lunar laser ranging). We also discuss the use of horizontal interferometers, including atom-chip or guided-atom devices, which potentially allow the use of longer coherence times in order to achieve higher sensitivity.

DOI: 10.1103/PhysRevD.80.016002

PACS numbers: 04.80.Cc, 03.75.Dg, 04.25.Nx, 11.30.Cp
\end{abstract}

\section{INTRODUCTION}

Local Lorentz invariance (LLI) in the gravitational interaction can be viewed as a prediction of the theory of general relativity. And it is not a trivial consequence, given that alternative theories of gravity have been put forward that do not lead to LLI, yet agree with general relativity in their predictions for the redshift, perihelion shift, and time delay. Experimental tests of the LLI in gravity are required to decide between these theories [1].

Another reason to perform tests of LLI in gravity is connected to one of the outstanding problems in physics, to find a unified theory of quantum gravity. The natural energy scale for such a theory is the Planck scale of about $10^{19} \mathrm{GeV}$. Direct experimentation at the Planck scale is, unfortunately, not possible. However, it is possible to search for suppressed effects at attainable energy scales in experiments of outstanding sensitivity. Violations of LLI ("Lorentz violation") are among the relatively few candidates for such observable consequences of quantum gravity [2].

The LLI of the nongravitational standard model has been tested for various particles, including photons [3-6], electrons [6-12], protons [8,10,13], neutrons [8-10,14],

*hm@berkeley.edu and others (see [2,15-18] for recent reviews). However, the LLI of gravity itself has been studied little to this date. Thus, it remains interesting and important to explore the validity of LLI in gravity.

For the theoretical descriptions of the consequences of Lorentz violation in gravity in the Solar System, a postNewtonian approximation is justified. Such descriptions are known as Nordtvedt's anisotropic universe model [19] or the standard model extension (SME) [20-22]; see Sec. IID for details. These frameworks use the following Lagrangian to describe the interaction between two pointmasses $M$ and $m$ [22]

$$
\begin{aligned}
\mathcal{L}= & \frac{1}{2} m v^{2}+G \frac{M m}{2 r}\left(2+3 \bar{s}^{00}+\bar{s}^{j k} \hat{r}^{j} \hat{r}^{k}-3 \bar{s}^{0 j} \boldsymbol{v}^{j}\right. \\
& \left.-\bar{s}^{0 j} \hat{r}^{j} \boldsymbol{v}^{k} \hat{r}^{k}\right) .
\end{aligned}
$$

For simplicity, we have taken $M$ to be at rest. We denote $\vec{r}$ the separation between $M$ and $m$, pointing towards $m$. The indices $j, k$ denote the spatial coordinates, $\vec{v}$ the relative velocity, and $\hat{r}=\vec{r} / r$. The components of $\bar{s}^{\mu \nu}=\bar{s}^{\nu \mu}$ specify Lorentz violation in gravity. If they vanish, LLI is valid.

The relative weakness of gravity means that only a small set of exceptionally sensitive experiments can place interesting limits on $\bar{s}$. Nordtvedt and Will noted that Lorentz violation in gravity would cause a modulation of the ap- 
parent local gravitational acceleration $g$ as the Earth rotates in space. Using gravimeter data [23] taken during the international geophysical year, July 1957-December 1958 , they obtained a limit of $\left|\bar{s}^{J K}\right| \leq 4 \times 10^{-9}(J, K \in$ $\{X, Y, Z\})$ on the spatial components $[1,19,24]$. More recently, Battat et al. analyzed 34 years of lunar laser ranging data, finding bounds on two linear combinations of the $\bar{s}^{J K}$ at a level of $\sim 10^{-10}$ [25]. They also found bounds on the three $\bar{s}^{T J}$ at levels of $\sim 10^{-7}$.

This means that for 4 degrees of freedom of $\bar{s}^{J K}$, there has been no improvement for about five decades. Moreover, a fundamental issue remains unaddressed: testing the isotropy of any force of nature means comparing it to another "standard" that is assumed to be isotropic. In other words, the isotropy of gravity can only be tested against the isotropy of another phenomenon, such as the velocity of light $c$. Past tests, however, are not easy to analyze in such depth as to make this comparison explicit. For example, it would be very difficult to analyze an influence of the isotropy of $c$ on the spring gravimeters used by Nordvedt and Will, although a description of Lorentz violation in solids is possible in principle [2628]. Hence, it is desirable to perform an experiment that relies on sufficiently simple physical principles so that all channels of influence for Lorentz violation can be theoretically described.

With atom interferometry [29-31], we have the unique situation of an experiment which is not only sensitive enough to obtain improved bounds on several elements of $\bar{s}^{J K}$, but also "clean" in the sense that we can quantitatively understand the influence of Lorentz violation in all relevant sectors. The basic principle is shown in Fig. 1: A matter wave packet is split by a beam splitter to form two interferometer arms. Because of the interaction with external potentials, such as gravity, the wave packet picks up a phase difference $\varphi$ between the arms. When the arms are recombined at a final beam splitter, $\varphi$ determines the probability that the atom is found emerging from one of the two interferometer outputs. Gravity's contribution to $\varphi$

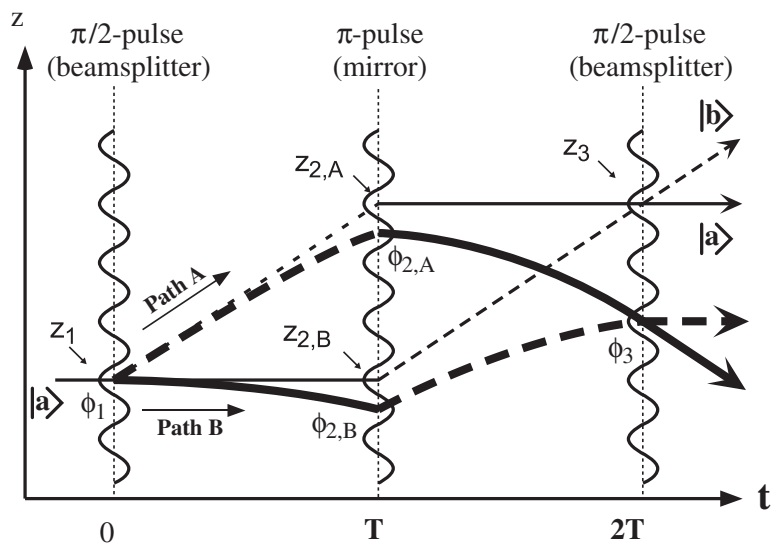

FIG. 1. Space-time diagram of a Mach-Zehnder atom interferometer. $t$ denotes time; $z$ denotes the vertical coordinate. can be several $10^{7}$ radians for the most sensitive devices [32,33].

Light-pulse atom interferometers use standing waves of laser light as gratings to diffract the matter waves; since the period of these gratings is given by the laser wavelength, the extremely high accuracy of laser frequency and phase stabilization can be applied to the measurement of gravity and other inertial forces.

Atom interferometers have been used for measurements of the fine-structure constant $\alpha$ [34-38], the local gravitational acceleration $g$ [39], its gradient [40], the Sagnac effect [41-43], or Newton's gravitational constant $[44,45]$. They rival or exceed the performance of other state-of-the-art methods. Thus, it seems natural to apply atom interferometry as a device to probe the weakest of the known forces of nature [30]. Detailed proposals have already been put forward, including tests of the Einstein equivalence principle [46] and detection of gravitational waves [47].

Here, we will discuss the first atom-interferometer test of post-Newtonian gravity [33]. It is also one of the few laboratory tests of gravity whose sensitivity is competitive with the best astrophysics data. This paper is organized as follows: We start with a description of the experiment in Sec. II; details not covered there will be found elsewhere $[33,39,48-50]$. Sections II A through IIC describe the setup; sec. IID describes the hypothetical signal for Lorentz violations in this experiment, including the influence of the electromagnetic sector. Section II E gives details of the data analysis. In Sec. III, we consider possibilities for future tests based on horizontal interferometers that may be based on atom chips [51-53] or other matter waveguides $[54,55]$.

\section{EXPERIMENT: A VERTICAL ATOMIC FOUNTAIN INTERFEROMETER}

\section{A. Principle}

In our Mach-Zehnder atom interferometer (Fig. 1), two vertical and antiparallel laser beams make the beam splitters and mirrors of the interferometer. They drive two photon Raman transitions: the atom absorbs a photon from one beam and is stimulated to emit a photon into the other one. Thus, the atom changes its hyperfine state and receives the momentum $2 \hbar k$ of two photons. A " $\pi / 2$ " pulse, which has its intensity and duration chosen such that this process happens with a probability of $1 / 2$, acts as a beam splitter; a " $\pi$ " pulse, with a transition probability close to 1 , makes a mirror.

For our Mach-Zehnder interferometer, a first $\pi / 2$ laser pulse transfers the atoms into a superposition of the $F=3$, $m_{F}=0$ and $F^{\prime}=4, m_{F}^{\prime}=0$ states. These move vertically relative to each other because of the momentum transferred by the laser radiation. A total of three light pulses split, reflect, and then recombine the paths to form an interfer- 
ometer. They are separated in time by the pulse separation time $T_{p}$.

The matter waves in both paths acquire a relative phase difference $\varphi=S_{C l} / \hbar+\varphi_{I}$ (see, e.g., Ref. [39] for details). The phase of the free evolution of the wave packet between the beam splitters is given by the classical action $S_{C l}$. If we restrict our attention to a constant gravitational acceleration $g$,

$$
\frac{S_{C l}}{\hbar}=\frac{1}{\hbar} \int_{0}^{2 T_{p}}\left[\frac{1}{2} m \dot{z}^{2}-m g z\right] d t .
$$

$z$ is the vertical coordinate and $m$ the atom's mass. By calculating the integral over the classical trajectories, it can be shown that this is the same for the upper and lower trajectory. Thus, it does not contribute to the phase difference between the interferometer arms.

The phase $\varphi_{I}$ is because whenever the atom changes state during an interaction, the phase of the atom changes by an amount equal to the phase of the light field. This adds phase when the atom absorbs a photon and subtracts phase when the atom emits one. Thus, it is easy to see that $\varphi_{I}=0$ for the trajectories without gravity, depicted by light lines in Fig. 1. Gravity accelerates the atoms downwards according to $\Delta z=-g t^{2} / 2$, as depicted by heavy lines. This gives rise to a phase shift of $k_{\text {eff }} \Delta z$, where $k_{\text {eff }}$ denotes the effective wave number. To high accuracy, the laser beams can be modeled as plane waves, which for two-photon transitions results in an effective wave number of $k_{\text {eff }}=$ $k_{1}+k_{2}$, where $k_{1,2}$ are the wave numbers of both lasers. For the upper trajectory, a photon pair is absorbed at $t=0$ and emitted at $t=T_{p}$. We can set the phase of that interaction to zero by definition. The phase of the second one will then be $k_{\mathrm{eff}} g T_{p}^{2} / 2$. For the lower path, a pair is absorbed at $t=T_{p}$ and one emitted at $2 T_{p}$. They add to a phase of $-k_{\text {eff }} g T_{p}^{2} / 2+k_{\text {eff }} g\left(2 T_{p}\right)^{2} / 2=3 k_{\text {eff }} g T_{p}^{2} / 2$. The difference for the two paths is thus $\varphi_{I}=k_{\mathrm{eff}} g T_{p}^{2}$. For the total phase shift, we take into account the possibility that the phases of the laser pulses at $z=0$ can experimentally be set arbitrary values $\varphi_{1}, \varphi_{2}, \varphi_{3}$ by suitable control of the laser system. We obtain [39]

$$
\varphi=k_{\mathrm{eff}} g T_{p}^{2}-\varphi_{L}, \quad \varphi_{L}=\varphi_{1}-2 \varphi_{2}+\varphi_{3}
$$

For our experiment, $T_{p}=0.4 \mathrm{~s}$ and $k \simeq 2 \pi /(852 \mathrm{~nm})$, so $\varphi \simeq 2.3 \times 10^{7} \mathrm{rad}$. In the experiment, we set $\varphi_{L}=r T_{p}^{2}$ by ramping the difference frequencies of the laser (actually, the continuous ramp can be approximated by discrete steps, as the laser pulse duration is very short). To measure $g$, the interferometer phase $\varphi$ is zeroed by adjusting the ramp rate to $r_{0} \approx 2 \pi \times 23 \mathrm{MHz} / \mathrm{s}$. This corresponds to finding the center of the interference pattern. Then, $g=$ $r_{0} /\left(k_{\text {eff }}\right)$. If $\varphi$ can be measured to, e.g., $1 \mathrm{mrad}$, we obtain a resolution of $10^{-10}$ in $g$.

\section{B. Fountain}

In our experimental setup (Fig. 2), we assemble about $10^{9} \mathrm{Cs}$ atoms within $650 \mathrm{~ms}$ from a background vapor pressure of $\sim 10^{-9}$ mbar in a three-dimensional magnetooptical trap (3D-MOT). A moving optical molasses launch accelerates them vertically upwards to a $\sim 1-\mathrm{s}$ ballistic trajectory with a temperature of $1.2-2 \mu \mathrm{K}$. Raman sideband cooling in a comoving optical lattice results in $\sim 3 \times$ $10^{8}$ atoms in the $F=3, m_{F}=3$ state at a (3D) temperature of $150 \mathrm{nK}$ that form a cloud of roughly $3 \mathrm{~mm}^{2}$ area [49]. A sudden change in the magnetic field followed by a $120-\mu$ s microwave pulse transfers $\sim 20 \%$ of them into the $F=4, m_{F}=0$ state. Atoms left over in the $F=3$ state are then cleared away using a resonant laser pulse. A solenoid generates a small magnetic bias field to set the quantization axis

\section{Interferometer}

The off resonant Raman pulses for the beam splitters have a wavelength of $852 \mathrm{~nm}$ and are generated by two grating-stabilized extended cavity diode lasers that are based on $100-\mathrm{mW}$ laser diodes SDL-5411. The first is frequency stabilized ("locked") to a cesium vapor cell using Doppler-free saturation spectroscopy. It arrives at the experiment with a detuning of $-1030 \mathrm{MHz}$ from the $6 S_{1 / 2}, F=3 \rightarrow 6 P_{3 / 2}, F^{\prime}=4$ transition in Cs. The second one is phase locked to the first one with a frequency difference close to the hyperfine splitting of $\simeq$ $9192 \mathrm{MHz}$, referenced to a LORAN-C frequency standard. The two lasers are overlapped on a beam splitter and the combined beam, containing $20 \mathrm{~mW}$ of each one, is transmitted to the experiment via a single-mode, polarization maintaining optical fiber. There, the beams are switched and intensity-controlled by an acousto-optical modulator (Isomet 1205). They are collimated with a $1 / e^{2}$ intensity diameter of about $2.5 \mathrm{~cm}$, and pass the vacuum chamber with linear polarization. Retro-reflection on top of it with two passes through a quarter-wave retardation plate forms a lin $\perp$ lin polarized counterpropagation geometry (Fig. 2).

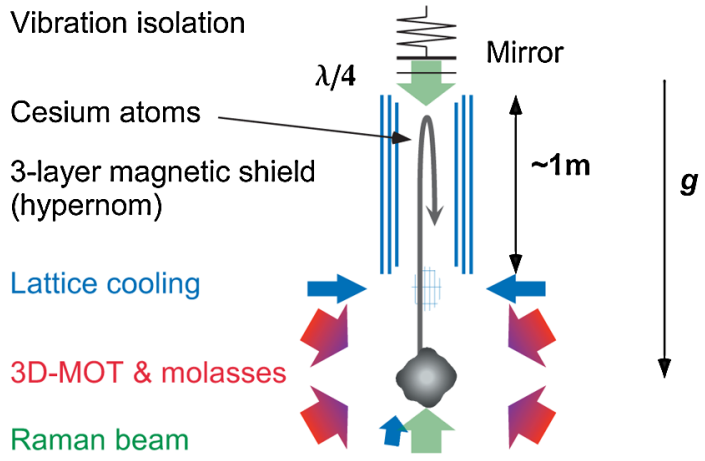

FIG. 2 (color online). Setup. $\lambda / 4 ; 1 / 4$-wave retardation plate. 
The error signal for the phase lock is generated by detecting the beat note after the fiber. Phase noise sources before this point can thus be taken out by the phase lock loop, whereas most others are common to both beams and do not affect the interferometer. The most important exception are residual vibrations of the top mirror, which we therefore reduce to below $5 \times 10^{-9} \mathrm{~g} / \sqrt{\mathrm{Hz}}$ in a frequency range of $0.1-10 \mathrm{~Hz}$ by a sophisticated active vibration isolator [48].

For fluorescence detection with a Hamamatsu R943-02 photomultiplier tube (PMT), the $F=4$ interferometer output is driven on a cycling transition; the $F=3$ output is detected subsequently after optical pumping to the $F=4$ state. Normalization of the signals takes out variations in the number of launched atoms.

Figure 3 shows a typical gravity fringe with a pulse separation time of $T_{p}=400 \mathrm{~ms}$, taken with 40 launches that take $75 \mathrm{~s}$ total. The sinewave fit has a phase uncertainty of $0.031 \mathrm{rad}$, and determines $g$ to an uncertainty of $\sim 1.3 \times$ $10^{-9} \mathrm{~g}$. This corresponds to $11 \times 10^{-9} \mathrm{~g} / \sqrt{\mathrm{Hz}}$. An improved short-term resolution of $8 \times 10^{-9} \mathrm{~g} / \sqrt{\mathrm{Hz}}$ can be reached by taking data at the $50 \%$ points of the fringes only. However, as this method is more sensitive to systematic effects such as drift of the PMT sensitivity [39], we used fringe fitting for taking the long term data. Our resolution is about 3 times better than the best previous one, which was reported by Peters et al. [39]. This is mainly a consequence of the increased interaction time $T=400 \mathrm{~ms}$. It also surpasses the best classical absolute gravimeter, the FG-5 falling corner cube gravimeter, by a factor of about 20.

\section{Hypothetical Signal}

The notion that the gravitational force between two objects might depend on the direction of the separation $\vec{r}$ could be described in very simple terms. For this work, however, we want to use a model that is as general as

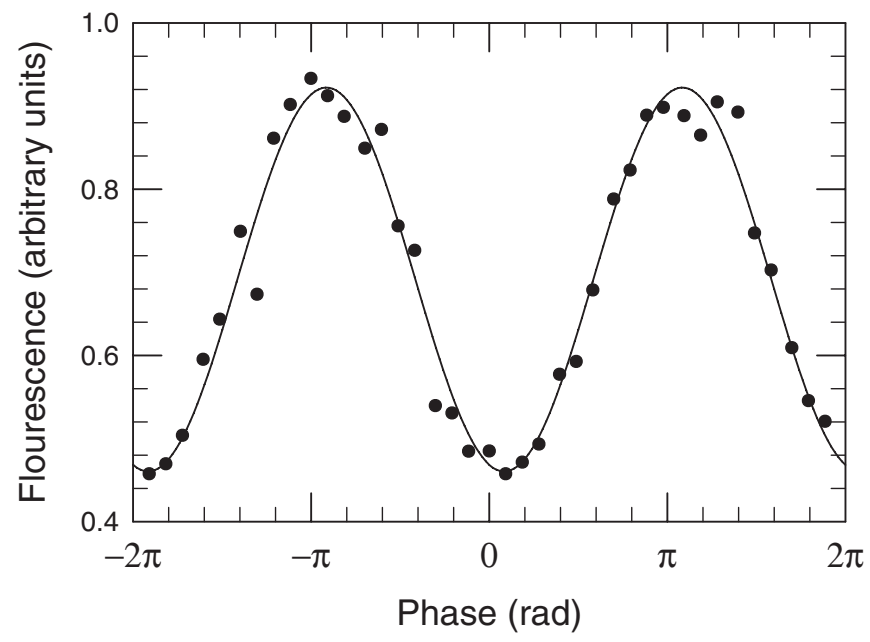

FIG. 3. Typical fringe obtained in our experiment. possible on the one hand and compatible with accepted principles that underlie the standard model and gravitational theory on the other hand. Two such models suggest themselves, Nordtvedt's anisotropic universe model [19] and the standard model extension [20-22] (a simplified formalism with one parameter has also been proposed [56]). The SME starts from a Lagrangian formulation of the standard model and gravity, adding general Lorentzviolating terms that can be formed from the fields and tensors. It is therefore a comprehensive theoretical framework that allows us to model violations of LLI in the various sectors of the standard model and gravity.

As $\varphi=k_{\text {eff }} g T_{p}^{2}$, the hypothetical signal for violations of LLI can be due to a change in $g$ and $k_{\text {eff }}$. The signal may thus be due to Lorentz violation in both the electromagnetic as well as the gravitational sector.

\section{Gravitational sector}

In a post-Newtonian approximation, the Lagrangian for the gravitational interaction in the SME is given by Eq. (1). The two-body Lagrangian of the anisotropic universe model is similar, but $\bar{s}^{00}=0$ and the coefficients of $v_{a}^{j}$ and $\hat{r}_{a b}^{j} \boldsymbol{v}_{a}^{k} \hat{r}_{a b}^{k}$ are independent of each other.

In principle, the components of $\bar{s}$ can be defined in any inertial frame of reference. For experiments on Earth (as well as on satellites), it is convenient to choose a Suncentered celestial equatorial reference frame [4]. It has the $X$ axis pointing towards the vernal equinox (spring point) at $0 \mathrm{~h}$ right ascension and $0^{\circ}$ declination, the $Z$ axis pointing towards the celestial north pole $\left(90^{\circ}\right.$ declination), and the $Y$ axis in the way needed to complete the right-handed orthogonal dreibein. Earth's equatorial plane is the $X-Y$ plane and the orbital plane of the Earth is tilted at an angle $\eta \simeq 23^{\circ}$ with respect to the latter. The time scale $T$ is set by $T=0$ when the Sun passes the spring point, which, for example, happened in 2001 on March 20, 13 h 31 min universal time (UT). Sun-centered frame quantities have Greek (0-3) or capital Latin indices.

We also define a laboratory frame, which has the $x$ axis pointing south, the $y$ axis east, and the $z$ axis vertically upwards. The laboratory time scale is set by $T_{\oplus}=0$ at any one instant when the $y$ and the $Y$ axis coincide. The difference between the two time scales $T_{\oplus}$ and $T$ can be written as a phase difference $\phi=\omega_{\oplus}\left(T_{\oplus}-T\right)$ [22], where $\omega_{\oplus}$ is the sidereal angular frequency of Earth's rotation; $\phi \simeq-1.77$ for this experiment. Laboratory-frame quantities have small Latin indices.

The derivation of the time-dependent modulations of $g$ for an observer on Earth involves taking into account the rotation and orbit of the Earth; the Earth itself is modeled as a massive sphere having a spherical moment of inertia of $I_{\oplus} \approx M_{\oplus} R_{\oplus}^{2} / 2$ [17] (not to be confused with the conventional moment of inertia, which for Earth is about $\left.M_{\oplus} r_{\oplus}^{2} / 3\right)$. It suffices to consider the first order in the Earth's orbital velocity $V_{\oplus} \simeq 10^{-4}$. Bailey and 
Kostelecky [22] have studied this in detail, and we refer the reader to this reference for the detailed signal components in the purely gravitational sector.

\section{Electromagnetic sector}

To study the variations of $k_{\text {eff }}$ caused by Lorentz violation, we start from the Lagrangian for the electromagnetic sector of the SME,

$$
\mathcal{L}=-\frac{1}{4} F^{\mu \nu} F_{\mu \nu}-\frac{1}{4}\left(k_{F}\right)_{\kappa \lambda \mu \nu} F^{\kappa \lambda} F^{\mu \nu},
$$

where $F^{\mu \nu}$ is the electromagnetic field tensor and $A^{\mu}$ the vector potential. The second term is proportional to a dimensionless tensor $\left(k_{F}\right)_{\kappa \lambda \mu \nu}$, which vanishes, if Lorentz invariance holds in electrodynamics. The tensor has 19 independent components [4]. A brief summary of the Maxwell equations that are derived from this Lagrangian is given in Appendix A.

For studying the Lorentz-violating modification to the effective wave vector $k_{\text {eff }}$ in the atom interferometer, the plane wave solutions have to be found. Making the standard ansatz $F_{\mu \nu}(x)=F_{\mu \nu}(p) e^{-i k_{\alpha} x^{\alpha}}$ for a plane wave with a wave 4-vector $k^{\alpha}=\left(k^{0}, \vec{k}\right)$ and inserting into Eq. (A1) one obtains the dispersion relation, that will allow us to determine $k_{\text {eff }}$ as a function of the direction of propagation. Let

$$
\begin{aligned}
& \rho=-\frac{1}{2} \tilde{k}_{\alpha}^{\alpha}, \quad \sigma^{2}=\frac{1}{2}\left(\tilde{k}_{\alpha \beta}\right)^{2}-\rho^{2}, \\
& \tilde{k}^{\alpha \beta}=\left(k_{F}\right)^{\alpha \mu \beta \nu} \hat{p}_{\mu} \hat{p}_{\nu}, \quad \hat{p}^{\mu}=\frac{p^{\mu}}{|\vec{p}|} .
\end{aligned}
$$

Then the dispersion relation is [4]

$$
k_{ \pm}^{0}=(1+\rho \pm \sigma)|\vec{k}| .
$$

The last term in this relation, which is proportional to $\sigma$, is purely polarization-dependent. Astrophysics shows that such a dependence, if it exists, is well below the levels relevant here [3]. We can thus assume $\sigma=0$.

To obtain the explicit time-dependence of the wave vector in our experiment, we need to transform the quantities, which are conventionally defined in a sun-centered celestial equatorial reference frame into the laboratory frame [4].

Explicit values of the Lorentz-violating quantities depend on the definition of coordinates and fields $[4,6,11,57]$. The freedom to define these can be used to set certain components to zero. A particular definition, for example, could be made by requiring $\tilde{\kappa}_{e-}^{J K}=0$ (see Appendix A). A choice like this would in general make these terms reappear in other sectors, such as the gravitational and fermionic sectors. In the following, we do not make any particular assumptions on such definitions and retain all the quantities $\bar{s}$ and $\tilde{\kappa}_{e-}, \tilde{\kappa}_{o+}$ in full generality.

\section{Combined signal}

Adding the contributions of the electromagnetic and the gravitational sector, the time dependence of the interferometer phase can be expressed as a Fourier series for the time dependence [22]

$$
\frac{\delta \varphi}{\varphi_{0}}=\sum_{m} C_{m} \cos \left(\omega_{m} t+\phi_{m}\right)+D_{m} \sin \left(\omega_{m} t+\phi_{m}\right) .
$$

The coefficients $C_{m}, D_{m}$ for the six frequencies $m \in$ $\left\{\omega_{\oplus}, 2 \omega_{\oplus}, \omega_{\oplus} \pm \Omega, 2 \omega_{\oplus} \pm \Omega\right\} \quad$ are functions of the Lorentz violations, that are given by the components of $\bar{s}^{\mu \nu}$ and $\left(k_{F}\right)_{\kappa \lambda \mu \nu}$ and the frequencies of Earth's orbit $\Omega_{\oplus}=2 \pi /(1 \mathrm{y})$ and rotation $\omega_{\oplus} \simeq 2 \pi /(23.93 \mathrm{~h})$. For a vertical interferometer, we obtain the signals for Lorentz violation in electromagnetism and gravity; see Table I. We denoted $i_{4}=1-3 I_{\oplus} /\left(M_{\oplus} R_{\oplus}^{2}\right) \approx-1 / 2$. The symbols $\tilde{\kappa}_{e-}, \tilde{\kappa}_{o+}$ denote linear combinations of the elements of $\left(k_{F}\right)_{\kappa \lambda \mu \nu}$ that are defined in Appendix A.

It turns out that for these components (but not, for example, for $C_{\Omega}, D_{\Omega}$ or the ones in Table VI), the substitutions

$$
i_{4} \sigma^{J K}=i_{4} \bar{s}^{J K}-\tilde{\kappa}_{e-}^{J K}, \quad i_{4} \sigma^{T J}=i_{4} \bar{s}^{T J}+\frac{1}{2} \epsilon_{J K L} \tilde{\kappa}_{o+}^{K L}
$$

can be used to obtain the combined signal components from the purely gravitational ones listed in Table IV of Ref. [22].

\section{E. Data analysis}

The combined data spans about $1500 \mathrm{~d}$, but fragmented

\begin{tabular}{|c|c|c|}
\hline Component & Amplitude & Phase \\
\hline$C_{2 \omega}$ & $\frac{1}{4} \sin ^{2} \chi\left[i_{4}\left(\bar{s}^{X X}-\bar{s}^{Y Y}\right)-\left(\tilde{\kappa}_{e-}^{X X}-\tilde{\kappa}_{e-}^{Y Y}\right)\right]$ & $2 \phi$ \\
\hline$D_{2 \omega}$ & $\frac{1}{2} \sin ^{2} \chi\left(i_{4} \bar{s}^{X Y}-\tilde{\kappa}_{e-}^{X Y}\right)$ & $2 \phi$ \\
\hline$C_{\omega}$ & $\frac{1}{2} \sin 2 \chi\left(i_{4} \bar{s}^{X Z}-\tilde{\kappa}_{e}^{X Z}\right)$ & $\phi$ \\
\hline$D_{\omega}$ & $\frac{1}{2} \sin 2 \chi\left(i_{4} \bar{s}^{Y Z}-\tilde{\kappa}_{e}^{Y Z}\right)$ & $\phi$ \\
\hline$C_{2 \omega+\Omega}$ & $-\frac{1}{4}(\cos \eta-1) V_{\oplus} \sin ^{2} \chi\left(i_{4} \bar{s}^{T Y}-\tilde{\kappa}_{o+}^{X Z}\right)$ & $2 \phi$ \\
\hline$D_{2 \omega+\Omega}$ & $\frac{1}{4}(\cos \eta-1) V_{\oplus} \sin ^{2} \chi\left(i_{4} \bar{s}^{T X}+\tilde{\kappa}_{o+}^{Y Z}\right)$ & $2 \phi$ \\
\hline$C_{2 \omega-\Omega}$ & $-\frac{1}{4}(\cos \eta+1) V_{\oplus} \sin ^{2} \chi\left(i_{4} \bar{s}^{T Y}-\tilde{\kappa}_{o+}^{X Z}\right)$ & $2 \phi$ \\
\hline$D_{2 \omega-\Omega}$ & $\frac{1}{4}(\cos \eta+1) V_{\oplus} \sin ^{2} \chi\left(i_{4} \bar{s}^{T X}+\tilde{\kappa}_{o+}^{Y Z}\right)$ & $2 \phi$ \\
\hline$C_{\omega+\Omega}$ & $\frac{1}{4} V_{\oplus} \sin \eta \sin ^{2} \chi\left(i_{4} \bar{s}^{T X}+\tilde{\kappa}_{o+}^{Y Z}\right)$ & $\phi$ \\
\hline$D_{\omega+\Omega}$ & $\begin{array}{c}\frac{1}{4} V_{\oplus}^{4} \sin ^{2} \chi\left[(1-\cos \eta)\left(i_{4} \bar{s}^{T Z}+\tilde{\kappa}_{o+}^{X Y}\right)\right. \\
\left.-\sin \eta\left(i_{4} \bar{s}^{T Y}-\tilde{\kappa}_{o+}^{X Z}\right)\right]\end{array}$ & $\phi$ \\
\hline$C_{\omega-\Omega}$ & $\frac{1}{4} V_{\oplus} \sin \eta \sin ^{2} \chi\left(i_{4} \bar{s}^{T X}+\tilde{\kappa}_{o+}^{Y Z}\right)$ & $\phi$ \\
\hline$D_{\omega-\Omega}$ & $\begin{array}{c}\frac{1}{4} V_{\oplus}^{4} \sin ^{2} \chi\left[(1+\cos \eta)\left(i_{4} \bar{s}^{T Z}+\tilde{\kappa}_{o+}^{X Y}\right)\right. \\
\left.+\sin \eta\left(i_{4} \bar{s}^{T Y}-\tilde{\kappa}_{o+}^{X Z}\right)\right]\end{array}$ & $\phi$ \\
\hline
\end{tabular}
into three relatively short segments: $\sim 60 \mathrm{~h}$ of data taken with this setup, as well as a $\sim 60 \mathrm{~h}$ and a $\sim 10 \mathrm{~d}$ run reported previously [39]; see Fig. 4.

TABLE I. Signal components for vertical atom interferometers. $\chi$ is geographical colatitude, $42.3^{\circ}$ for Stanford. 


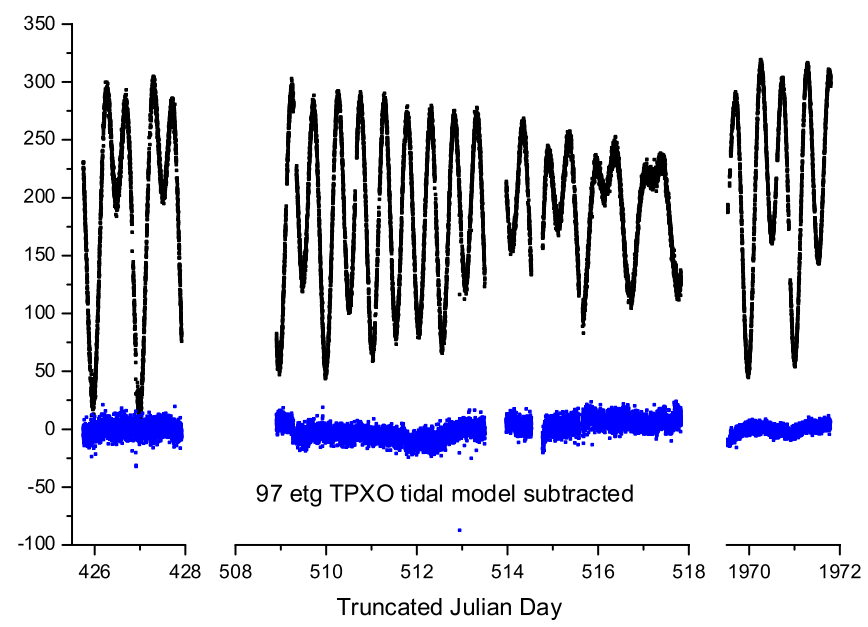

FIG. 4 (color online). Data in $10^{-9}$ g. Each point represents a 60-s scan of one fringe (75-s after TJD1900).

Major systematic effects in this experiment are tidal variations of the local gravitational acceleration. Subtraction of a Newtonian model of these influences [58] based on the relative positions of the Sun, the Moon, and the planets, yields the residues shown at the bottom of Fig. 4. In addition to the tidal model used in our paper, we here use an additional model of the local tides [59]. This will allow us to significantly reduce the relatively large estimates on some of the $\sigma$ coefficients reported in the paper. As our old analysis and analyses using lunar laser ranging [25], the present analysis is based on an assumption of no accidental cancellations of signals, i.e., that the model does not contain the same influence of Lorentz violation as our measurement.

In order to extract the signal components of Table I, we calculate the discrete Fourier transform. As is typical of a finite set of data, the Fourier components overlap. This overlap can be quantified by a covariance matrix. For a compact presentation, let

$$
\begin{gathered}
\omega_{m}=\left(2 \omega_{\oplus}, \omega_{\oplus}, 2 \omega_{\oplus}+\Omega, 2 \omega_{\oplus}-\Omega, \omega_{\oplus}+\Omega, \omega_{\oplus}-\Omega\right), \\
a_{2 m+1}=\cos \left(\omega_{m} t+\phi_{m}\right), \quad a_{2 m}=\sin \left(\omega_{m} t+\phi_{m}\right) .
\end{gathered}
$$

For a total of $K$ data, let $d\left(t_{k}\right), k \in\{1, \ldots K\}$ be the datum taken at the time $t_{k}$. The sine and cosine Fourier transforms are

$$
\tilde{d}_{m}=\frac{2}{K} \sum_{k} d\left(t_{k}\right) a_{m}\left(t_{k}\right) .
$$

The $\tilde{d}_{m}$ that are obtained from the fragmented data are a linear combination

$$
\tilde{d}_{m}=\sum_{n} \operatorname{cov}\left(a_{m}, a_{n}\right) \tilde{D}_{n}
$$

of the corresponding Fourier components $\tilde{D}_{m}$ for hypothetical continuous data. The covariance matrix

$$
\operatorname{cov}\left(a_{m}, a_{n}\right)=\frac{2}{K} \sum_{k} a_{m}\left(t_{k}\right) a_{n}\left(t_{k}\right)
$$

where the sum is over all $t_{k}$. For data that span a large time scale, it approaches a unit matrix, i.e., the overlap of the Fourier coefficients becomes negligible. The covariance matrix for our data is shown in Table II.

Multiplication of the covariance matrix (Table II) with the signals listed in Table I [written as a vector $\left.\left(C_{2 \omega}, D_{2 \omega}, C_{\omega}, \ldots, D_{\omega-\Omega}\right)\right]$ gives the linear combinations that the experiment is sensitive to; see Table III. For obtaining bounds on Lorentz violation, we perform a numerical Fourier analysis of the data for the 12 components $c_{m}, d_{m}$; see the table. The error is estimated by performing a Fourier analysis at several frequencies above and below the signal frequencies and computing the root of the mean square.

The limits listed in Table III are on linear combinations of parameters. The 12 results are sufficient to determine all 7 parameters. In order to obtain independent estimates for the parameters while making optimum use of the experimental data, we proceed as follows. Each datum corresponds to a probability distribution $p_{n}\left(\sigma^{T X}, \ldots, \sigma^{Y Z}\right)$, where $n=1, \ldots 12$, that we assume to be Gaussian with the center and standard deviation as tabulated. Multiplying all 12 distributions results in one overall probability distribution $P\left(\sigma^{T X}, \ldots, \sigma^{Y Z}\right)=A \prod_{n=1}^{12} p_{n}$, where $A$ is a normalization factor. In order, for example, to obtain an estimate on $\sigma^{T X}$ that is independent of the other coefficients, we integrate over the other variables, $P\left(\sigma^{T X}\right)=$ $A^{\prime} \int_{-\infty}^{\infty} d \sigma^{T Y} \ldots \int_{-\infty}^{\infty} d \sigma^{Y Z} P\left(\sigma^{T X}, \ldots, \sigma^{Y Z}\right)$ (where all variables but $\sigma^{T X}$ are integrated over and $A^{\prime}$ is another normalization factor). The result is a probability distribution for $\sigma^{T X}$ that has one maximum and standard deviation, which are our estimate and error bar for this coefficient. Specifically, since we assumed the $p_{n}$ to be Gaussian, $P\left(\sigma^{T X}\right)$ will be a Gaussian and the most probable estimate as well as one $\sigma$ error are read off.

Table IV shows the results thus obtained. Compared to the ones that we published in our paper [33] (derived from the same data), the limits are now more uniform: whereas

TABLE II. Correlation matrix cov.

\begin{tabular}{lllllllllllll}
\hline \hline 1 & 0 & 0 & 0 & 0.3 & -0.6 & 0.3 & 0.5 & 0 & 0 & 0.1 & 0 \\
0 & 1 & 0 & 0 & 0.6 & 0.3 & -0.6 & 0.3 & 0 & 0 & 0 & 0 \\
0 & 0 & 1 & 0 & 0 & -0.1 & 0 & 0.1 & 0.6 & -0.6 & 0.6 & 0.6 \\
0 & 0 & 0 & 1 & 0 & 0 & 0 & 0 & 0.6 & 0.6 & -0.6 & 0.6 \\
0.3 & 0.6 & 0 & 0 & 1 & 0 & -0.6 & 0.7 & 0 & 0 & 0 & 0 \\
-0.6 & 0.3 & -0.1 & 0 & 0 & 1 & -0.7 & -0.6 & 0 & 0 & 0 & 0 \\
0.3 & -0.6 & 0 & 0 & -0.6 & -0.7 & 1 & 0 & 0 & 0 & 0 & 0 \\
0.5 & 0.3 & 0.1 & 0 & $0.7-0.6$ & 0 & 1 & 0 & -0.1 & 0 & 0 \\
0 & 0 & 0.6 & 0.6 & 0 & 0 & 0 & 0 & 1 & 0 & 0.3 & 0.6 \\
0 & 0 & -0.6 & 0.6 & 0 & 0 & 0 & -0.1 & 0 & 1 & -0.6 & 0.3 \\
0.1 & 0 & 0.6 & -0.6 & 0 & 0 & 0 & 0 & 0.3 & -0.6 & 1 & 0 \\
0 & 0 & 0.6 & 0.6 & 0 & 0 & 0 & 0 & 0.6 & 0.3 & 0 & 1 \\
\hline \hline
\end{tabular}


TABLE III. Signal components as obtained by a numerical Fourier transform of the data. They correspond to linear combinations of the components of $\sigma$, with linear coefficients as tabulated. The result of the Fourier transform is listed as fraction of $g$.

\begin{tabular}{llllllllc}
\hline \hline Component & \multicolumn{1}{c}{$\sigma^{T X}$} & \multicolumn{1}{c}{$\sigma^{T Y}$} & $\sigma^{T Z}$ & $\sigma^{X X}-\sigma^{Y Y}$ & $\sigma^{X Y}$ & $\sigma^{X Z}$ & $\sigma^{Y Z}$ & Measurement/10 \\
\hline$c_{2 \omega}$ & -0.091 & 0.040 & -0.002 & -0.079 & 0.004 & -0.009 & 0.004 & $-0.097 \pm 0.24$ \\
$d_{2 \omega}$ & -0.039 & -0.094 & -0.007 & 0.001 & -0.241 & -0.007 & 0.001 & $0.009 \pm 0.24$ \\
$c_{\omega}$ & -0.066 & 0.001 & -0.142 & -0.003 & -0.007 & -0.240 & 0.001 & $0.69 \pm 0.27$ \\
$d_{\omega}$ & 0.003 & -0.063 & -0.136 & 0.001 & 0.001 & 0.001 & -0.243 & $-0.034 \pm 0.27$ \\
$c_{2 \omega+\Omega}$ & -0.105 & -0.094 & -0.005 & -0.020 & -0.141 & -0.007 & -0.004 & $0.19 \pm 0.24$ \\
$d_{2 \omega+\Omega}$ & 0.098 & -0.107 & 0.011 & 0.044 & -0.068 & 0.016 & -0.003 & $-0.22 \pm 0.24$ \\
$c_{2 \omega-\Omega}$ & -0.005 & 0.158 & -0.001 & -0.021 & 0.144 & -0.002 & 0.006 & $-0.15 \pm 0.24$ \\
$d_{2 \omega-\Omega}$ & -0.157 & -0.000 & -0.004 & -0.043 & -0.064 & -0.014 & 0.006 & $0.40 \pm 0.24$ \\
$c_{\omega+\Omega}$ & -0.065 & -0.034 & -0.131 & -0.003 & -0.002 & -0.140 & -0.143 & $0.20 \pm 0.27$ \\
$d_{\omega+\Omega}$ & 0.036 & -0.064 & -0.053 & 0.003 & 0.004 & 0.139 & -0.151 & $-0.73 \pm 0.27$ \\
$c_{\omega-\Omega}$ & -0.067 & 0.029 & -0.003 & -0.005 & -0.001 & -0.143 & 0.140 & $0.28 \pm 0.27$ \\
$c_{\omega-\Omega}$ & -0.029 & -0.061 & -0.231 & -0.001 & -0.007 & -0.142 & -0.148 & $0.25 \pm 0.27$ \\
\hline \hline
\end{tabular}

the best limits on the three $\sigma^{T J}$ and four (combinations of) $\sigma^{J K}$ are of the same order, the worst ones from the new analysis are about 4 times better than in the paper. This is a result of our use of a more sophisticated tidal model as well as an optimum method to derive individual limits from the limits on linear combinations.

\section{F. Combination with lunar laser ranging (LLR)}

Our experiment thus provides the only measurement of the components of $\sigma$ so far; these are combinations of gravitational and electromagnetic Lorentz violation. To compare our data with previous experiments, which have not been analyzed for the electromagnetic influence (although such an influence exist), we here adopt the assumption that there is no Lorentz violation in electromagnetism, i.e., $\tilde{\kappa}_{e-}^{J K}=\tilde{\kappa}_{o+}^{J K}=0$. Our limits (Table IV) then correspond directly to bounds on $\bar{s}$.

Lunar laser ranging [25] bounds two linear combinations of $\bar{s}^{J K}[25]$

$$
\begin{aligned}
\bar{s}^{11}-\bar{s}^{22} \equiv & 0.08\left(\bar{s}^{X X}+\bar{s}^{Y Y}-2 \bar{s}^{Z Z}\right)-0.31\left(\bar{s}^{X X}-\bar{s}^{Y Y}\right) \\
& -1.7 \bar{s}^{X Y}+0.60 \bar{s}^{X Z}+0.42 \bar{s}^{Y Z} \\
= & (1.3 \pm 0.9) \times 10^{-10}, \\
\bar{s}^{12} \equiv & 0.43\left(\bar{s}^{X X}-\bar{s}^{Y Y}\right)-0.31 \bar{s}^{X Y}-0.23 \bar{s}^{X Z}-0.33 \bar{s}^{Y Z} \\
= & (6.9 \pm 4.5) \times 10^{-11} .
\end{aligned}
$$

TABLE IV. Atom-interferometry limits on Lorentz violation in gravity and electrodynamics.

\begin{tabular}{lc}
\hline \hline Coefficient & \\
\hline$\sigma^{T X}$ & $(-3.1 \pm 5.1) \times 10^{-5}$ \\
$\sigma^{T Y}$ & $(0.1 \pm 5.4) \times 10^{-5}$ \\
$\sigma^{T Z}$ & $(1.4 \pm 6.6) \times 10^{-5}$ \\
$\sigma^{X X}-\sigma^{Y Y}$ & $(4.4 \pm 11) \times 10^{-9}$ \\
$\sigma^{X Y}$ & $(0.2 \pm 3.9) \times 10^{-9}$ \\
$\sigma^{X Z}$ & $(-2.6 \pm 4.4) \times 10^{-9}$ \\
$\sigma^{Y Z}$ & $(-0.3 \pm 4.5) \times 10^{-9}$ \\
\hline
\end{tabular}

They can be combined with our results from Table III (using the method described above) to obtain independent limits on one more degree of freedom of $\bar{s}^{J K}$. Battat et al. also report four limits on the three $\bar{s}^{T J}$, [25]

$$
\begin{aligned}
\bar{s}^{01} & =-0.60 \bar{s}^{T X}+0.82 \bar{s}^{\underline{\underline{T Y}}}(-0.8 \pm 1.1) \times 10^{-6}, \\
\bar{s}^{02} & =-0.53 \bar{s}^{T Y}-0.75 \bar{s}^{T X}+0.40 \bar{s}^{T Z} \\
& =(-5.2 \pm 4.8) \times 10^{-7} \\
\bar{s}_{\Omega_{\oplus} C} & =-3.1 \bar{s}^{T Y}-1.1 \bar{s}^{T Z}+0.094 \bar{s}^{T X} \\
& =(0.2 \pm 3.9) \times 10^{-7} \\
\bar{s}_{\Omega_{\oplus} S} & =-3.4 \bar{s}^{T X}+0.037 \bar{s}^{T Y}+0.15 \bar{s}^{T Z} \\
& =(-1.3 \pm 4.1) \times 10^{-7} .
\end{aligned}
$$

They can be combined with ours to increase the resolution of the limits.

Table V lists the results thus obtained. They represent the most complete bounds on Lorentz violation in gravity, providing individual limits on the $\bar{s}$ as well as more components of $\bar{s}$ and higher resolution than either experiment. The only degrees of freedom of $\bar{s}^{J K}$ that are not bounded are $\bar{s}^{T T}$ and the trace, which do not lead to signals to first order in the Earth's orbital velocity.

TABLE V. Bounds resulting from combining our data with the ones from lunar laser ranging as reported by Battat et al. [25], assuming vanishing Lorentz violation in electrodynamics.

\begin{tabular}{lc}
\hline \hline Coefficient & \\
\hline $\bar{s}^{T X}$ & $(0.5 \pm 6.2) \times 10^{-7}$ \\
$\bar{s}^{T Y}$ & $(0.1 \pm 1.3) \times 10^{-6}$ \\
$\bar{s}^{T Z}$ & $(-0.4 \pm 3.8) \times 10^{-6}$ \\
$\bar{s}^{X X}-\bar{s}^{Y Y}$ & $(-1.2 \pm 1.6) \times 10^{-9}$ \\
$\bar{s}^{X X}+\bar{s}^{Y Y}-2 \bar{s}^{Z Z}$ & $(1.8 \pm 38) \times 10^{-9}$ \\
$\bar{s}^{X Y}$ & $(-0.6 \pm 1.5) \times 10^{-9}$ \\
$\bar{s}^{X Z}$ & $(-2.7 \pm 1.4) \times 10^{-9}$ \\
$\bar{s}^{Y Z}$ & $(0.6 \pm 1.4) \times 10^{-9}$ \\
\hline \hline
\end{tabular}




\section{SIGNAL FOR HORIZONTAL INTERFEROMETERS}

In this section, we consider tests of gravity with horizontal atom interferometers, including guided-atom devices. Testing LLI in gravity is a task that makes good use of the features of such interferometers, in particular, long coherence times and hence high resolution. Moreover, since the signal for violations is a time-dependent modulation, the stability of the interferometer on time scales much larger than the modulation frequencies are not a primary concern for such tests.

For simplicity, we shall again assume a vanishing of Lorentz violation in electrodynamics throughout this section.

A test of the LLI of gravity can be performed by measuring a Lorentz-violating horizontal acceleration. These accelerations are given by [22]

$a^{x}=-g i_{3} \bar{s}^{x z}-\omega_{\oplus}^{2} R_{\oplus} \sin \chi \cos \chi+g i_{3} \bar{s}^{T z}+g i_{3} \bar{s}^{T x} V_{\oplus}^{z}$,

$a^{y}=-g i_{3} \bar{s}^{y z}+g i_{3} \bar{s}^{T z}+g i_{3} \bar{s}^{T y} V_{\oplus}^{z}$,

where $i_{3}=1-I_{\oplus} /\left(M_{\oplus} R_{\oplus}^{2}\right) \approx 1 / 2$. For the purpose of this section, we can take $a^{z}=g$ as well as $a \equiv|a| \approx g$ to be constant. Such tests can, for example, be based on a torsion pendulum, which is suspended off its center of mass. Nevertheless, they might reach superior sensitivity compared to vertical gravimeters such as the atom interferometer discussed previously.

Compared to a conventional torsion pendulum, such experiments involve special challenges associated with maintaining the pendulum within the horizontal plane. However, measurement of the horizontal accelerations that are given by Eqs. (15) with atom interferometry is possible using conventional horizontal interferometers. Moreover, interferometers in the horizontal plane can be built well using atom-chip or atomic waveguide techniques. In contrast to atomic fountains, they allow long pulse separation times $T_{p}$ in a compact setup. It is therefore interesting to study the signals for Lorentz violation in post-Newtonian gravity and electromagnetism for such an interferometer.

We assume a horizontal Mach-Zehnder interferometer with the laser beams pointing into a direction of

$$
\hat{x}=(\cos \theta, \sin \theta, 0)
$$

in the laboratory frame. As before, the phase shift is given by $k_{\text {eff }} T_{p}^{2}(\hat{x} \cdot \vec{g})$, where the local gravitational acceleration $\vec{g}$ has vertical as well as horizontal components.

The calculation of the induced time dependence of the interferometer phase proceeds via the transformations between the laboratory frame and the Sun-centered standard frame. The fastest way to do this is probably by analogy to the case of torsion balances that has been considered in [22]; see Appendix B. After all, both measure the accelerations given by Eqs. (15). As a result, we can express the contribution of Lorentz violation in gravity to the phase as

$$
\begin{aligned}
\varphi= & k_{\mathrm{eff}} i_{3} g T_{p}^{2} \sum_{n}\left(\left[E_{n} \sin \alpha_{n}-F_{n} \cos \alpha_{n}\right] \sin \omega_{n} T\right. \\
& \left.-\left[E_{n} \cos \alpha_{n}+F_{n} \sin \alpha_{n}\right] \cos \omega_{n} T\right) .
\end{aligned}
$$

The amplitudes and phases in this expression are given in Table VI. It is evident that horizontal interferometers provide access to four independent linear combinations of $\bar{s}^{J K}$ (the same ones as vertical interferometers) and sufficient data to determine all the $\bar{s}^{T J}$.

\section{SUMMARY AND OUTLOOK}

In this paper, we have presented atom-interferometry tests of the local Lorentz invariance of post-Newtonian gravity and electrodynamics. As a comprehensive and quantitative model for violations, we use the standard model extension $[20,21]$. The relevant violations of LLI in gravity are encoded by a tensor $\bar{s}$ [22]; those for violations in electromagnetism are expressed by the matrices $\tilde{\kappa}_{e-}$ and $\tilde{\kappa}_{o+}$ [3]. The experimental signal for Lorentz violation is a time dependence of the local gravitational acceleration as the Earth orbits in the solar system. We discuss an experiment that has been performed by us [33] as well as possible future experiments with horizontal interferometer geometries.

Our experiment is a vertical Mach-Zehnder atom interferometer. It uses a bright source of cesium atoms in a 1-m high atomic fountain and a pulse separation time of $400 \mathrm{~ms}$. A resolution of up to $8 \times 10^{-9} \mathrm{~g} / \sqrt{\mathrm{Hz}}$ is reached, the highest of any cold-atom gravimeter so far.

For this experiment, the signal for Lorentz violation is given by a particular combination of coefficients entering the gravitational and electromagnetic sectors: $\sigma^{J K}=$ $\bar{s}^{J K}-\tilde{\kappa}_{e-}^{J K} / i_{4}$ and $\sigma^{T J}=\bar{s}^{T J}+\epsilon_{J K L} \tilde{\kappa}_{o+}^{K L} /\left(2 i_{4}\right)$. Here, $i_{4} \simeq$ $-1 / 2$ is given by the Earth's spherical moment of inertia.

For the analysis, we use about $2.5 \mathrm{~d}$ of data taken with the setup just described as well as about $12.5 \mathrm{~d}$ of data taken with a previous setup [39]. The data have been taken over a total time interval of about $1500 \mathrm{~d}$. A major systematic effect are the tides, which we account for by subtracting a Newtonian model that is based on the relative positions of the Earth, the Moon, the Sun, and planets. By Fourier analyzing the residuals at different combinations of the frequencies of Earth's rotation and orbit, we obtain bounds on 12 linear combinations of seven components of $\sigma^{J K}$ at the $10^{-9}$ level.

Our limits are the strongest bounds on several combinations of coefficients for Lorentz violation, even in view of previous work from geophysical gravity observations [19] and lunar laser ranging [25]. This makes our experiment one of the few competitive laboratory tests of gravity. Moreover, our experiment is presently the only test of Lorentz invariance of gravity that explicitly takes into account the possibility of Lorentz violation in the non- 
gravitational standard model. This is important when interpreting the results in the context of the search for quantum-gravity signals.

The physics behind atom interferometry and lunar laser ranging is similar, monitoring the trajectory of a proof mass within Earth's gravitational field by laser beams. However, they differ in the orbit (if one can think of the atoms' trajectory as an orbit) and quantum-mechanical nature of the mass. Indeed, the equivalence principle for quantum objects has been discussed [60]. Also, ours is a laboratory experiment, which typically offers superior control over the experimental systematics. Moreover, it is so far the only experiment where the simultaneous influence of the nongravitational and gravitational effects are understood quantitatively and which accordingly states combined bounds.

Assuming that Lorentz invariance in electrodynamics vanishes, the data from LLR and our experiment can be combined to yield improved and more detailed bounds.

We also studied the signals for Lorentz violation (in gravity and electromagnetism) for horizontal interferometers. They are attractive for this type of measurement, as they offer increased coherence time in a compact setup.

Taking several months worth of data would help to eliminate the dominant lunar tides, the major systematic effect. With a data set that spans a year, even the leading solar tides could be suppressed.

Future work may lead to alternative bounds from torsion balances, gravimetry data that is routinely taken in geophysical research, or an analysis of data from the gravity probe-B satellite [61]. Note, however, that our results are still not limited by any fundamental influence such as quantum projection noise. With typically $10^{8}$ atoms per launch, a quantum projection limited gravimeter could reach the $10^{-12} \mathrm{~g}$ level per launch and $10^{-14} \mathrm{~g}$ per day, if other noise sources (notably phase noise and vibrations) can be controlled. Also, the performance of atom interferometers can be increased further by utilizing beam splitters that transfer the momentum of many photons, thus leading to sensitivity increases by factors of 12 (demonstrated) to 100 times (anticipated) [38,62,63]. This promises improved tests of gravity based on atom interferometry, deepening our understanding of the fundamental principles of nature.

\section{ACKNOWLEDGMENTS}

We thank A. Peters and A. Senger for valuable help and important discussions.

\section{APPENDIX A: ELECTROMAGNETIC SECTOR}

The Maxwell equations in vacuum that are derived from the Lagrangian, Eq. (4), are

$$
\partial_{\alpha} F_{\mu}^{\alpha}+\left(k_{F}\right)_{\mu \alpha \beta \gamma} \partial^{\alpha} F^{\beta \gamma}=0, \quad \partial_{\mu} \tilde{F}^{\mu \nu}=0,
$$

where

$$
\tilde{F}^{\mu \nu}=\frac{1}{2} \varepsilon^{\mu \nu \alpha \beta} F_{\alpha \beta} .
$$

They can be written in analogy to the Maxwell equations in anisotropic media [4]: With the $3 \times 3$ matrices

$$
\begin{array}{ll}
\left(\kappa_{D E}\right)^{j k}=-2\left(k_{F}\right)^{0 j 0 k}, \quad\left(\kappa_{H B}\right)^{j k}=\frac{1}{2} \epsilon^{j p q} \epsilon^{k r s}\left(k_{F}\right)^{p q r s}, \\
\left(\kappa_{D B}\right)^{j k}=\left(k_{F}\right)^{0 j p q} \epsilon^{k p q}, \quad\left(\kappa_{H E}\right)^{k j}=-\left(\kappa_{D B}\right)^{j k}
\end{array}
$$

(Latin indices take the values 1,2,3) one can define $\vec{D}$ and $\vec{H}$ fields

$$
\left(\begin{array}{c}
\vec{D} \\
\vec{H}
\end{array}\right)=\left(\begin{array}{cc}
\mathbb{1}+\kappa_{D E} & \kappa_{D B} \\
\kappa_{H E} & \mathbb{1}+\kappa_{H B}
\end{array}\right)\left(\begin{array}{c}
\vec{E} \\
\vec{B}
\end{array}\right)
$$

where $\mathbb{1}$ represents the $3 \times 3$ unit matrix. The Maxwell equations can now be expressed as [4]

$$
\begin{array}{ll}
\vec{\nabla} \times \vec{H}-\partial_{0} \vec{D}=0, & \vec{\nabla} \cdot \vec{B}=0, \\
\vec{\nabla} \times \vec{E}+\partial_{0} \vec{B}=0, & \vec{\nabla} \cdot \vec{D}=0 .
\end{array}
$$

Lorentz violation in electrodynamics is thus analogous to electrodynamics in anisotropic media. For later use, we define the linear combinations

$$
\begin{aligned}
\left(\tilde{\kappa}_{e+}\right)^{j k} & =\frac{1}{2}\left(\kappa_{D E}+\kappa_{H B}\right)^{j k}, \\
\left(\tilde{\kappa}_{o+}\right)^{j k} & =\frac{1}{2}\left(\kappa_{D B}+\kappa_{H E}\right)^{j k}, \\
\left(\tilde{\kappa}_{e^{-}}\right)^{j k} & =\frac{1}{2}\left(\kappa_{D E}-\kappa_{H B}\right)^{j k}-\frac{1}{3} \delta^{j k}\left(\kappa_{D E}\right)^{l l}, \\
\left(\tilde{\kappa}_{o^{-}}\right)^{j k} & =\frac{1}{2}\left(\kappa_{D B}-\kappa_{H E}\right)^{j k}, \quad \tilde{\kappa}_{t r}=\frac{1}{3}\left(\kappa_{D E}\right)^{l l} .
\end{aligned}
$$

Of these, the 10 degrees of freedom of $\tilde{\kappa}_{o-}$ and $\tilde{\kappa}_{e+}$ encode birefringence; they are bounded to below $10^{-37}$ by observations of gamma-ray bursts [3,4]. The residual nine cause a dependence of the velocity of light on the direction of propagation or, vice versa, such a dependence of the wave number of light having a constant frequency. They are therefore relevant in interferometry experiments.

\section{APPENDIX B: ANALOGY OF HORIZONTAL INTERFEROMETERS AND TORSION PENDULUM EXPERIMENTS}

The free oscillations of a torsion pendulum are governed by the differential equation $\ddot{\vartheta}+(\kappa / I) \vartheta=\tau$, where $I$ is the moment of inertia, and $\tau$ represents the driving force. It can be expressed by an effective potential $V$ as $\tau=$ $-d V / d \vartheta$. In the limit of $\kappa \rightarrow \infty$, we simply have

$$
\theta(T)=-\frac{1}{\kappa} \frac{d V}{d \vartheta}
$$

Kostelecky and Bailey [22] give the oscillations $\vartheta(T)$ by 
TABLE VI. Amplitude and phase for the gravitational part of the signal in horizontal interferometers.

\begin{tabular}{|c|c|c|}
\hline & Amplitude & $\alpha_{n}$ \\
\hline$E_{2 \omega}$ & $-\bar{s}^{X Y} \sin \theta \sin \chi-\frac{1}{4}\left(\bar{s}^{X X}-\bar{s}^{Y Y}\right) \cos \theta \sin 2 \chi$ & $2 \phi$ \\
\hline$F_{2 \omega}$ & $\frac{1}{2}\left(\bar{s}^{X X}-\bar{s}^{Y Y}\right) \sin \theta \sin \chi-\frac{1}{2} \bar{s}^{X Y} \cos \theta \sin 2 \chi$ & $2 \phi$ \\
\hline$E_{\omega}$ & $-\bar{s}^{Y Z} \sin \theta \cos \chi-\bar{s}^{X Z} \cos \theta \cos 2 \chi$ & $\phi$ \\
\hline$F_{\omega}$ & $\bar{s}^{X Z} \sin \theta \cos \chi-\bar{s}^{Z Y} \cos \theta \cos 2 \chi$ & $\phi$ \\
\hline$E_{2 \omega+\Omega}$ & $\frac{1}{2} V_{\oplus} \bar{s}^{T X} \sin \theta(1-\cos \eta) \sin \chi-\frac{1}{4} V_{\oplus} \bar{s}^{T Y} \cos \theta(1-\cos \eta) \sin 2 \chi$ & $2 \phi$ \\
\hline$F_{2 \omega+\Omega}$ & $\frac{1}{2} V_{\oplus} \bar{s}^{T Y} \sin \theta(1-\cos \eta) \sin \chi+\frac{1}{4} V_{\oplus} \bar{s}^{T X} \cos \theta(1-\cos \eta) \sin 2 \chi$ & $2 \phi$ \\
\hline$E_{2 \omega-\Omega}$ & $-\frac{1}{2} V_{\oplus} \bar{s}^{T X} \sin \theta(1+\cos \eta) \sin \chi+\frac{1}{4} V_{\oplus} \bar{s}^{T Y} \cos \theta(1+\cos \eta) \sin 2 \chi$ & $2 \phi$ \\
\hline$F_{2 \omega-\Omega}$ & $-\frac{1}{2} V_{\oplus} \bar{s}^{T Y} \sin \theta(1+\cos \eta) \sin \chi-\frac{1}{4} V_{\oplus} \bar{s}^{T X} \cos \theta(1+\cos \eta) \sin 2 \chi$ & $2 \phi$ \\
\hline$E_{\omega+\Omega}$ & $\begin{array}{c}-\frac{1}{2} V_{\oplus} \bar{s}^{T Y} \sin \theta \sin \eta \cos \chi+V_{\oplus}^{4} \bar{s}^{T X} \cos \theta \sin \eta\left(\frac{1}{2}-\cos ^{2} \chi\right) \\
-\frac{1}{2} V_{\oplus} \bar{s}^{T Z} \sin \theta(\cos \eta-1) \cos \chi\end{array}$ & $\phi$ \\
\hline$F_{\omega+\Omega}$ & $\begin{array}{c}\frac{1}{2} V_{\oplus} \bar{s}^{T X} \sin \theta \sin \eta \cos \chi-V_{\oplus} \bar{s}^{T Z} \cos \theta(1-\cos \eta)\left(\frac{1}{2}-\cos ^{2} \chi\right) \\
+V_{\oplus} \bar{s}^{T Y} \cos \theta \sin \eta\left(\frac{1}{2}-\cos ^{2} \chi\right)\end{array}$ & $\phi$ \\
\hline$E_{\omega-\Omega}$ & $\begin{array}{c}-\frac{1}{2} V_{\oplus} \bar{s}^{T Z} \sin \theta(1+\cos \eta) \cos \chi+\frac{1}{2} V_{\oplus} \bar{s}^{T Y} \sin \theta \sin \eta \cos \chi \\
+V_{\oplus} \bar{s}^{T X} \cos \theta \sin \eta\left(\frac{1}{2}-\cos ^{2} \chi\right)\end{array}$ & $\phi$ \\
\hline$F_{\omega-\Omega}$ & $\begin{array}{c}\frac{1}{2} V_{\oplus} \bar{s}^{T X} \sin \theta \sin \eta \cos \chi+V_{\oplus} \bar{s}^{T Y} \cos \theta \sin \eta\left(\frac{1}{2}-\cos ^{2} \chi\right) \\
+V_{\oplus} \bar{s}^{T Z} \cos \theta(1+\cos \eta)\left(\frac{1}{2}-\cos ^{2} \chi\right)\end{array}$ & $\phi$ \\
\hline$E_{\Omega}$ & $-\frac{1}{2} V_{\oplus} \bar{s}^{T Y} \cos \theta \cos \eta \sin 2 \chi+V_{\oplus} \bar{s}^{T Z} \cos \theta \sin \eta \sin 2 \chi$ & 0 \\
\hline$F_{\Omega}$ & $\frac{1}{2} V_{\oplus} \bar{s}^{T X} \cos \theta \sin 2 \chi$ & 0 \\
\hline
\end{tabular}

their Eq. (134). For small excitations $\vartheta$,

$$
\frac{d V}{d \vartheta}=-m r_{0}\left(a^{x} S_{N}-a^{y} C_{N}+\frac{1}{2} a \omega_{\oplus}^{2} \sin ^{2} \chi S_{N 2}\right) .
$$

Equating this with Bailey and Kostelecky’s Eq. (134) in the limit of $\omega_{0} \rightarrow \infty$, we obtain

$$
\begin{aligned}
a^{x} S_{N}- & a^{y} C_{N}+\frac{1}{2} a \omega_{\oplus}^{2} \sin ^{2} \chi S_{N 2} \\
= & i_{3} g \sum_{n}\left(\left[E_{n} \sin \alpha_{n}-F_{n} \cos \alpha_{n}\right] \sin \left(\omega_{n} T\right)\right. \\
& \left.-\left[E_{n} \cos \alpha_{n}+F_{n} \sin \alpha_{n}\right] \cos \left(\omega_{n} T\right)\right),
\end{aligned}
$$

where the amplitudes $E_{n}, F_{n}$ and the phases $\alpha_{n}$ are tabulated in Table $\mathrm{V}$ of [22]. They represent the time dependence of the Lorentz-violating horizontal accelerations as functions of the Earth's orbital parameters and the $C_{N}, S_{N}$, $S_{2 N}$. Specializing to the case of one mass, $C_{N}=\cos \theta$, $S_{N}=\sin \theta$, and $S_{2 N}=\sin 2 \theta$. By comparison of coefficients, we obtain explicit expressions for $a_{x}, a_{y}$, and $a_{z}$ in the laboratory frame, that we can insert into the equation $\varphi=\vec{k}_{\text {eff }} \vec{g} T_{p}^{2}$ for the interferometer's phase. Note that with our specification of the atom interferometer's orientation $\hat{x}$, Eq. (16), $\theta=\vartheta+\pi / 2$, which has been taken into account in Table VI.
[1] C. M. Will, Astrophys. J. 169, 141 (1971); Living Rev. Relativity 4, 4 (2001)

[2] G. Amelino-Camelia, C. Lämmerzahl, A. Macias, and H. Müller, AIP Conf. Proc. 758, 30 (2005).

[3] V. A. Kostelecký and M. Mewes, Phys. Rev. Lett. 97, 140401 (2006); 99, 011601 (2007).

[4] V. A. Kostelecký and M. Mewes, Phys. Rev. D 66, 056005 (2002).

[5] S. M. Carroll, G. B. Field, and R. Jackiw, Phys. Rev. D 41, 1231 (1990); R. Bluhm, V. A. Kostelecký, and C. D. Lane, Phys. Rev. Lett. 84, 1098 (2000); V. W. Hughes et al., ibid. 87, 111804 (2001); J. A. Lipa, J. A. Nissen, S. Wang, D. A. Stricker, and D. Avaloff, ibid. 90, 060403 (2003); H. Müller, S. Herrmann, C. Braxmaier, S. Schiller, and A. Peters, ibid. 91, 020401 (2003); P. Wolf, S. Bize, A. Clairon, G. Santarelli, M.E. Tobar, and A. N. Luiten, Phys. Rev. D 70, 051902(R) (2004); P. L. Stanwix et al.,
Phys. Rev. Lett. 95, 040404 (2005); S. Herrmann, A. Senger, E. Kovalchuk, H. Müller, and A. Peters, ibid. 95, 150401 (2005); M. E. Tobar, P. Wolf, A. Fowler, and J. G. Hartnett, Phys. Rev. D 71, 025004 (2005); P. Antonini, M. Okhapkin, E. Göklü, and S. Schiller, Phys. Rev. A 72, 066102 (2005); P. L. Stanwix, M. E. Tobar, P. Wolf, C.R. Locke, and E. N. Ivanov, Phys. Rev. D 74, 081101(R) (2006); C.D. Carone, M. Sher, and M. Vanderhaeghen, Phys. Rev. D 74, 077901 (2006); B. Feng, M. Li, J. Q. Xia, X. Chen, and X. Zhang, Phys. Rev. Lett. 96, 221302 (2006); S. Reinhardt et al., Nature Phys. 3, 861 (2007); M. Hohensee, A. Glenday, C.-H. Li, M.E. Tobar, and P. Wolf, Phys. Rev. D 75, 049902(E) (2007); F. R. Klinkhamer and M. Risse, ibid. 77, 016002 (2008).

[6] H. Müller et al., Phys. Rev. Lett. 99, 050401 (2007).

[7] R. K. Mittleman, I. I. Ioannou, H. G. Dehmelt, and N. 
Russell, Phys. Rev. Lett. 83, 2116 (1999); H. Dehmelt, R. Mittleman, R. S. VanDyck, and P. Schwinberg, Phys. Rev. Lett. 83, 4694 (1999); L.-S. Hou, W. T. Ni, and Y.-C. M. Li, Phys. Rev. Lett. 90, 201101 (2003); B. R. Heckel et al., Phys. Rev. Lett. 97, 021603 (2006); B. Altschul, Phys. Rev. D 74, 083003 (2006); 75, 041301(R) (2007).

[8] T. W. Kornack, G. Vasilakis, and M. V. Romalis, in CPT and Lorentz Symmetry IV, edited by V. A. Kostelecký (World Scientific, Singapore, 2008).

[9] L. Hunter et al., in CPT and Lorentz Symmetry I, edited by V. A. Kostelecký (World Scientific, Singapore, 1999).

[10] V. A. Kostelecky and C. D. Lane, Phys. Rev. D 60, 116010 (1999).

[11] H. Müller, S. Herrmann, A. Saenz, A. Peters, and C. Lämmerzahl, Phys. Rev. D 68, 116006 (2003).

[12] C. D. Lane, Phys. Rev. D 72, 016005 (2005).

[13] G. Gabrielse et al., Phys. Rev. Lett. 82, 3198 (1999); D. F. Phillips, M. A. Humphrey, E. M. Mattison, R. E. Stoner, R. F. C. Vessot, and R. L. Walsworth, Phys. Rev. D 63, 111101 (2001); M. A. Humphrey et al., Phys. Rev. A 68, 063807 (2003); P. Wolf, F. Chapelet, S. Bize, and A. Clairon, Phys. Rev. Lett. 96, 060801 (2006).

[14] D. Bear, R. E. Stoner, R. L. Walsworth, V. A. Kostelecky, and C. D. Lane, Phys. Rev. Lett. 85, 5038 (2000); F. Cané et al., ibid. 93, 230801 (2004); B. Altschul, Phys. Rev. D 75, 023001 (2007).

[15] D. Mattingly, Living Rev. Relativity 8, 5 (2005).

[16] C. M. Will, Living Rev. Relativity 9, 3 (2006)

[17] C.M. Will, Theory and Experiment in Gravitational Physics (Cambridge University Press, Cambridge, England, 1993).

[18] V. A. Kostelecky and N. Russell, arXiv:0801.0287.

[19] K. Nordvedt, Phys. Rev. D14, 1511 (1976).

[20] D. Colladay and V. A. Kostelecky, Phys. Rev. D 55, 6760 (1997); 58, 116002 (1998).

[21] V. A. Kostelecky, Phys. Rev. D 69, 105009 (2004).

[22] Q. G. Bailey and V.A. Kostelecky, Phys. Rev. D 74, 045001 (2006).

[23] J. C. Harrison, N. F. Ness, I. M. Longman, R. F. S. Forbes, E. A. Kraut, and L. B. Slichter, J. Geophys. Res. 68, 1497 (1963).

[24] K. Nordtvedt and C. M. Will, Astrophys. J. 177, 775 (1972).

[25] J. B. R. Battat, J. F. Chandler, and C. W. Stubbs, Phys. Rev. Lett. 99, 241103 (2007).

[26] H. Müller, S. Herrmann, A. Saenz, A. Peters, and C. Lämmerzahl, Phys. Rev. D 68, 116006 (2003).

[27] H. Müller, Phys. Rev. D 71, 045004 (2005).

[28] H. Müller, S. Herrmann, A. Saenz, A. Peters, and C. Lämmerzahl, Phys. Rev. D 70, 076004 (2004).

[29] A.D. Cronin, J. Schmiedmayer, and D. E. Pritchard, arXiv:0712.3703v1 [Rev. Mod. Phys. (to be published)].

[30] M. Kasevich and S. Chu, Phys. Rev. Lett. 67, 181 (1991).

[31] S. Chu, Nature (London) 416, 206 (2002).

[32] J. Le Gouët, T. Mehlstäubler, J. Kim, Sébastien Merlet, A. Clairon, A. Landragin, and F.P. Dos Santos, arXiv:0801.1270v1.

[33] H. Müller, S.-w. Chiow, S. Herrmann, S. Chu, and K.-Y. Chung, Phys. Rev. Lett. 100, 031101 (2008).

[34] D. S. Weiss, B. C. Young, and S. Chu, Phys. Rev. Lett. 70, 2706 (1993); M. Weitz, B. C. Young, and S. Chu, ibid. 73,
2563 (1994).

[35] A. Wicht et al., Phys. Scr. T102, 82 (2002).

[36] P. Cladé et al., Phys. Rev. Lett. 96, 033001 (2006); Phys. Rev. A 74, 052109 (2006).

[37] H. Müller et al., Appl. Phys. B 84, 633 (2006).

[38] H. Müller, S.-w. Chiow, Q. Long, S. Herrmann, and S. Chu, Phys. Rev. Lett. 100, 180405 (2008).

[39] A. Peters, K. Y. Chung, and S. Chu, Nature (London) 400, 849 (1999); Metrologia 38, 25 (2001).

[40] M. J. Snadden, J. M. McGuirk, P. Bouyer, K. G. Haritos, and M. A. Kasevich, Phys. Rev. Lett. 81, 971 (1998).

[41] T.L. Gustavson, A. Landragin, and M.A. Kasevich, Classical Quantum Gravity 17, 2385 (2000).

[42] D. S. Durfee, Y. K. Shaham, and M. A. Kasevich, Phys. Rev. Lett. 97, 240801 (2006).

[43] B. Canuel, F. Leduc, D. Holleville, A. Gauguet, J. Fils, A. Virdis, A. Clairon, N. Dimarcq, Ch. J. Borde, A. Landragin, and P. Bouyer, Phys. Rev. Lett. 97, 010402 (2006).

[44] J. B. Fixler et al., Science 315, 74 (2007).

[45] G. Lamporesi, A. Bertoldi, L. Cacciapuoti, M. Prevedelli, and G. M. Tino, Phys. Rev. Lett. 100, 050801 (2008).

[46] S. Dimopoulos, P. W. Graham, J. M. Hogan, and M. A. Kasevich, Phys. Rev. Lett. 98, 111102 (2007); Phys. Rev. D 78, 042003 (2008).

[47] S. Dimopoulos, P. W. Graham, J. M. Hogan, M. A. Kasevich, and S. Rajendran, Phys. Rev. D 78, 122002 (2008).

[48] J. M. Hensley, A. Peters, and S. Chu, Rev. Sci. Instrum. 70, 2735 (1999).

[49] P. Treutlein, K. Y. Chung, and S. Chu, Phys. Rev. A 63, 051401(R) (2001).

[50] K. Chung, Ph.D. thesis, Stanford University, 2001.

[51] S. Hofferberth, I. Lesanovsky, B. Fischer, J. Verdu, and J. Schmiedmayer, Nature Phys. 2, 710 (2006).

[52] Y.-J. Wang, D.Z. Anderson, V. M. Bright, E. A. Cornell, Q. Diot, T. Kishimoto, M. Prentiss, R. A. Saravanan, S. R. Segal, and S. Wu, Phys. Rev. Lett. 94, 090405 (2005).

[53] Y. Shin, C. Sanner, G.-B. Jo, T. A. Pasquini, M. Saba, W. Ketterle, D. E. Pritchard, M. Vengalattore, and M. Prentiss, Phys. Rev. A 72, 021604(R) (2005).

[54] S. Wu, E. J. Su, and M. Prentiss, Phys. Rev. Lett. 99, 173201 (2007).

[55] S. Gupta, K. W. Murch, K. L. Moore, T. P. Purdy, and D. M. Stamper-Kurn, Phys. Rev. Lett. 95, 143201 (2005).

[56] M. Li, Y. Pang, and Y. Wang, arXiv:0903.0227v2 [Phys. Rev. D (to be published)].

[57] H. Müller, S. Herrmann, A. Senger, E. Kovalchuk, A. Peters, P.L. Stanwix, M.E. Tobar, E. Ivanov, and P. Wolf, in CPT and Lorentz. Symmetry IV, edited by A Kostelecký (World Scientific, Singapore, 2008), p. 302.

[58] Calculated from the Tamura [64] potentials using the ETGTAB V3.0 solid earth tide data generation software by H. G. Wenzel, Universität Karlsruhe. See, for example, http://www.geo.uni-jena.de/geophysik/etc/etcdat/etgtab/.

[59] G. D. Egbert, A. Bennett, and M.G. G. Foreman, J. Geophys. Res. 99, 24821 (1994).

[60] A. Herdegen and J. Wawrzycki, Phys. Rev. D 66, 044007 (2002).

[61] J. Overduin, in CPT and Lorentz Symmetry IV, edited by A Kostelecký (World Scientific, Singapore, 2008), p. 199. 
[62] S. Herrmann, S.-w. Chiow, S. Chu, and H. Müller, arXiv:0901.1819.

[63] H. Müller, S.-w. Chiow, S. Herrmann, and S. Chu, arXiv:0903.4192 [Phys. Rev. Lett. (to be published)].
[64] Y. Tamura, Bulletin d'Information Marées Terrestres, edited by P. Melchior (Observatoire Royal de Belgique, Brussels, 1987), Vol. 99, p. 6813. 